\title{
VLTI/AMBER spectro-interferometric imaging of VX Sagittarii's inhomogenous outer atmosphere ${ }^{\star}$
}

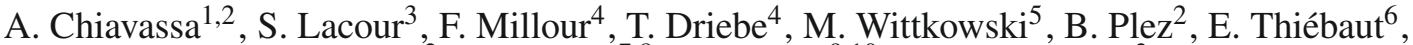

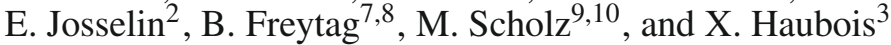

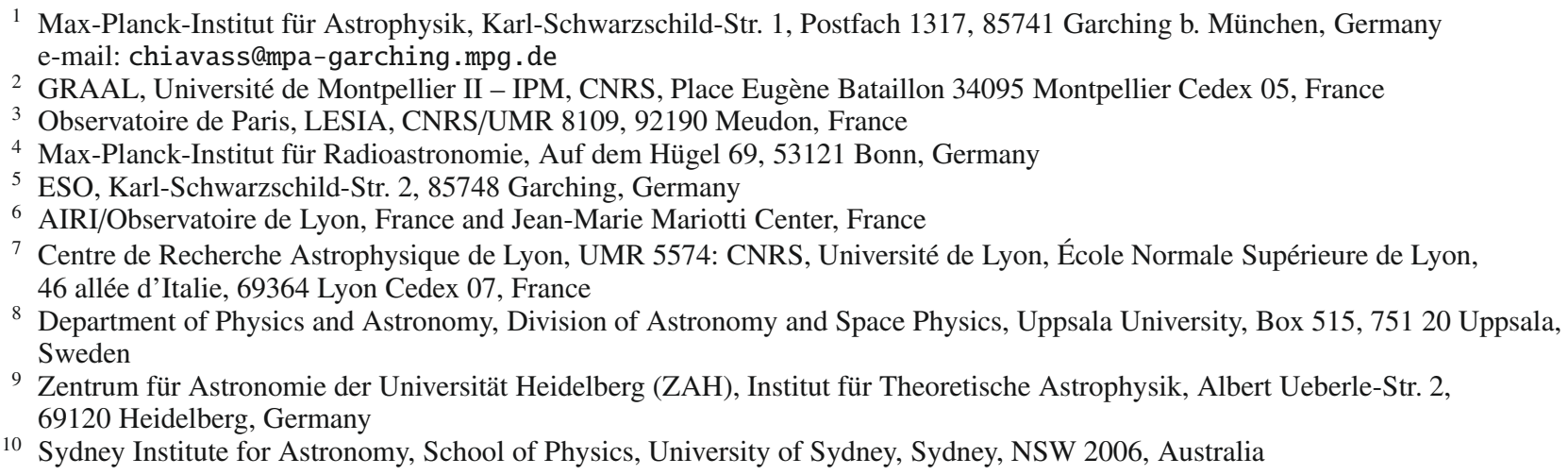

Received 13 September 2009 / Accepted 23 November 2009

\section{ABSTRACT}

\begin{abstract}
Aims. We aim to explore the photosphere of the very cool late-type star VX Sgr and in particular the characterization of molecular layers above the continuum forming photosphere.

Methods. We obtained interferometric observations with the VLTI/AMBER interferometer using the fringe tracker FINITO in the spectral domain $1.45-2.50 \mu \mathrm{m}$ with a spectral resolution of $\approx 35$ and baselines ranging from 15 to $88 \mathrm{~m}$. We performed independent image reconstruction for different wavelength bins and fit the interferometric data with a geometrical toy model. We also compared the data to 1D dynamical models of Miras atmosphere and to 3D hydrodynamical simulations of red supergiant (RSG) and asymptotic giant branch (AGB) stars.

Results. Reconstructed images and visibilities show a strong wavelength dependence. The $H$-band images display two bright spots whose positions are confirmed by the geometrical toy model. The inhomogeneities are qualitatively predicted by 3D simulations. At $\approx 2.00 \mu \mathrm{m}$ and in the region $2.35-2.50 \mu \mathrm{m}$, the photosphere appears extended and the radius is larger than in the $H$ band. In this spectral region, the geometrical toy model locates a third bright spot outside the photosphere that can be a feature of the molecular layers. The wavelength dependence of the visibility can be qualitatively explained by $1 \mathrm{D}$ dynamical models of Mira atmospheres. The best-fitting photospheric models show a good match with the observed visibilities and give a photospheric diameter of $\Theta=8.82 \pm$ 0.50 mas. The $\mathrm{H}_{2} \mathrm{O}$ molecule seems to be the dominant absorber in the molecular layers.

Conclusions. We show that the atmosphere of VX Sgr seems to resemble Mira/AGB star model atmospheres more closely than do RSG model atmospheres. In particular, we see molecular (water) layers that are typical of Mira stars.
\end{abstract}

Key words. stars: AGB and post-AGB - stars: atmospheres - stars: individual: VX Sagittarii - techniques: interferometric supergiants

\section{Introduction}

VX Sagittarii (HD 165674) is a cool semi-regular variable with a long mean period of 732 days (Kholopov et al. 1987). Lockwood \& Wing (1982) reports a spectral type varying from M5.5 (near the time of visual maximum) to M9.8 (at minimum light). Lockwood \& Wing (1982) determined that the effective temperature of VX Sgr ranges between 3300 and $2400 \mathrm{~K}$ (maximum to minimum light). García-Hernández et al. (2007) find $T_{\text {eff }}=2900 \mathrm{~K}$ at the time of their high-resolution spectroscopic observation, when the star was near minimum light $\left(\mathrm{AAVSO}^{1}\right)$.

* Based on the observations made with VLTI-ESO Paranal, Chile under the programs IDs 081.D-0005(A, B, C, D, E, F, G, H).

1 www. aavso.org
Lockwood \& Wing also find that VX Sgr exhibits stronger CN and VO bands than to Mira variables with similar temperature. Enhanced $\mathrm{CN}$ absorption is an indicator of high luminosity in RSGs of an earlier type and also, together with VO, of S stars. Speck et al. (2000) categorized VX Sgr as an oxygenrich star and found a strong silicate feature at $10 \mu \mathrm{m}$, indicates a dusty circumstellar environment. Using aperture-masking and IR/optical-telescope array interferometry at $2.16 \mu \mathrm{m}$, Monnier et al. (2004) reveals that VX Sgr exhibits a dusty environment with a flux contribution of about $20 \%$ in the $K$ band and some evidence of departure from circular symmetry, even if they could not place strong limits on possible asymmetries because of calibration uncertainties. The dusty environment is confirmed by HST images (Schuster et al. 2006). VX Sgr's circumstellar 
environment is the result of the heavy mass loss experienced by the star $\left(1.3 \times 10^{-5} M_{\odot} \mathrm{yr}^{-1}, \mathrm{CO}\right.$ measurements; Knapp et al. 1989). The mass-loss process appears to be particularly asymmetric for the inner regions (Chapman \& Cohen 1986). Using AAVSO data, Kamohara et al. (2005) show that the optical light curve has a much smaller amplitude of about 2 mag in the years 1998-2003, much less than the usual 6-7 mag. An examination of AAVSO data shows that the decrease to this smaller amplitude has happened several times in the past 70 years and that the star is probably currently in that state.

The classification of VX Sgr as a red supergiant or an AGB is thus not firmly established. A further constraint can be brought by an estimate of its luminosity, in order to better ascertain its position in the HR diagram. Humphreys et al. (1972) placed VX Sgr in the vicinity of the Sgr OB1 cluster at $1.7 \mathrm{kpc}$; Murakawa et al. (2003) find $1.8 \pm 0.5 \mathrm{kpc}$ measuring the $\mathrm{GHz}$ $\mathrm{H}_{2} \mathrm{O}$ maser expansion; Chen et al. (2007) reports a distance of $1.57 \pm 0.27 \mathrm{kpc}$ using $43 \mathrm{GHz} \mathrm{SiO}$ maser proper motions; finally, the trigonometric parallax of Hipparcos (van Leeuwen 2007) gives a distance of $0.262(+0.655 /-0.109) \mathrm{kpc}$, which is probably unreliable because of the size and asymmetry of the stellar photosphere.

Using AAVSO data, we find VX Sgr was at maximum luminosity during our observations, and we assume a $T_{\text {eff }}$ of 3200 to $3400 \mathrm{~K}$. With the 2MASS $K$ magnitude (Cutri et al. 2003), assuming a distance $d=1.7 \mathrm{kpc}$ and using data for Galactic red supergiants from Levesque et al. (2005), we derive a luminosity $\log L / L_{\odot}=5.25 \pm 0.25\left(M_{\text {bol }}=-8.4 \pm 0.6\right)$. The error bar accounts for uncertainties in the photometry and in the assumed $T_{\text {eff }}$ at the time of our observation, affecting the bolometric correction at $K$. Circumstellar emission in IR may increase the luminosity by a few tenths of magnitude. Putting the star at $1.3 \mathrm{kpc}$ would decrease the estimated luminosity to $\log L / L_{\odot}=$ $5.00 \pm 0.25$. The radius is then about $1200 R_{\odot}$, and $A_{\mathrm{v}}=2$ to 4 . This is a too high luminosity for an AGB star (e.g., Vassiliadis $\&$ Wood 1993). Even compared to so-called super-AGB stars, where the most recent models show a maximum of $\log L / L_{\odot} \approx$ 4.8 with typical masses ranging between $\approx 7-11 M_{\odot}$ (Siess 2006) and $\log L / L_{\odot} \approx 5$ (Poelarends et al. 2008), VX Sgr's luminosity is extremely high. García-Hernández et al. (2009) find AGB stars with similar luminosities and masses of $\approx 6-7 M_{\odot}$ showing $\mathrm{Rb}$ enhancement in the Magellanic Clouds, and they argue that these AGB stars may be more luminous due to a contribution from hot bottom burning. However, García-Hernández et al. (2007), using synthetic spectra based on classical hydrostatic model atmospheres for cool stars with extensive line lists, find VX Sgr to be the only Li-poor, long-period, high $\mathrm{OH}$ expansion velocity star of their Galactic AGB sample. On the other hand, VX Sgr's low effective temperature and large V variability are quite atypical for RSGs, although Levesque et al. (2007) find high-variability, low- $T_{\text {eff }}$ RSGs in the Magellanic Clouds. Heger et al. (1997) studied the pulsations properties in red supergiants from 10 to $20 M_{\odot}$ with a high luminosity-to-mass ratio and show that very large pulsation periods, amplitudes, and mass-loss rates may be expected to occur at and beyond central helium ex-haustion over the time scale of the last few $10^{4}$ years. This could lead to an overall dimming of the star after a period of stronger oscillations subsequent to enhanced mass loss and ejection of a dust shell that screens the stellar radiation. It appears that the evolutionary status of VX Sgr is still not well established, more investigations are needed. In particular its chemical composition should be scrutinized.

We discuss here interferometric observations of VX Sgr made with the VLTI/AMBER instrument in the near IR. The aim of this paper is to study the continuum-forming photosphere, and the characterization of molecular layers of VX Sgr probing different wavelengths in the $H$ and $K$ bands.

\section{Observations and data reduction}

We obtained near-infrared interferometry data of VX Sgr with the Very Large Telescope Interferometer (VLTI, Haguenauer et al. 2008) using the near-infrared beam combiner AMBER (Petrov et al. 2007), which simultaneously covers the $J, H$, and $K$ bands with a spectral resolution of $\approx 35$. VX Sgr has been observed in less than 1.5 months using the AT configurations: A0-D0-H0, D0-H0-G1, and E0-G0-H0. The fringe tracker FINITO (Le Bouquin et al. 2008) was used for all the observations. In addition to the science target, three calibrator stars were observed close in time and interleaved with VX Sgr: HD 169916 (K0IV), HD 146545 (K5III), and HD 166295 (K2III/IV). The calibrator diameters were retrieved from Richichi et al. (2005), Bordé et al. (2002), and Richichi \& Percheron (2002). The diameter errors are about $1 \%$. Details are reported in Table 1 with the projected baseline lengths $\left(B_{\mathrm{p}}\right)$, the position angles $\left(\mathrm{PA}_{\mathrm{p}}\right)$, the spectral interval, the calibrators used, the optical seeing, and the coherence time.

Raw visibilities and closure phases were computed with the latest version of the amdlib package (release 2.2, Tatulli et al. 2007) and the yorick interface provided by the Jean-Marie Mariotti Center. Individual frames were averaged after selecting the $20 \%$ best frames based only on the fringe SNR and with a piston smaller than $\pm 20 \mu \mathrm{m}$. We decided to discard $J$ band fringes because the data quality was significantly worse than for longer wavelengths.

In addition, we used the addons by Millour et al. (2008, release 1.53) to calibrate the datasets. The transfer function measurement for one night is shown in Fig. 1. Both science and calibrator targets have the same detector integration time of $0.05 \mathrm{~ms}$, and after the calibration, VX Sgr data were averaged. The error bars on the calibrated visibilities include the statistical error of averaging the single frames, the errors of the calibration stars' angular diameters, and the scatter of the transfer function measurements. This scatter (top panel of Fig. 1) is much larger than the internal errors, computed by the reduction software. This basically means that the visibilities errors (between 0.05 and 0.1 ) we use in this paper reflect calibration issues simultaneously affecting a whole range of wavelengths, while the wavelengthto-wavelength error is much smaller (typically 0.01 to 0.05 ). While the visibility errors seem large in a single dataset, the wavelength-variation errors of the same dataset are small.

The absolute wavelength correction was done using the telluric Kitt Peak spectra, which we convolved to match the spectral resolution of the AMBER data. In VX Sgr data, the band gaps (i.e., between $J$ and $H$ and $H$ and $K$ ) are visible, and we made a linear two-component adjustment of the wavelength scale, which gave a systematic offset of $-0.21 \mu \mathrm{m}$ with respect to the initial AMBER table and a 7\% wavelength stretch.

Figure 2 shows the final UV-plane coverage of all observations that successfully passed all steps of the data reduction and calibration quality control. The north-west south-east direction is not completely covered because of the actual AT geometry, while the east-west direction is particularly favored. The star exhibits high wavelength-dependent visibilities and has a clear non-zero, non- $180^{\circ}$ closure phase (bottom panel of Fig. 1), showing asymmetries in the intensity distribution. 
A. Chiavassa et al.: VLTI/AMBER spectro-interferometric imaging of VX Sagittarii's inhomogenous outer atmosphere

Table 1. Observations log for the AMBER observations of VX Sgr, carried out using FINITO and an integration time of 50 ms.

\begin{tabular}{|c|c|c|c|c|c|c|}
\hline Date & $\begin{array}{l}B_{\mathrm{p}} \\
{[\mathrm{m}]}\end{array}$ & $\begin{array}{c}\mathrm{PA}_{\mathrm{p}} \\
{\left[{ }^{\circ}\right]}\end{array}$ & $\begin{array}{l}\text { Spectral } \\
\text { range }[\mu \mathrm{m}]\end{array}$ & Calibrators & $\begin{array}{c}\text { Seeing } \\
{\left[{ }^{\prime \prime}\right]}\end{array}$ & $\begin{array}{c}\text { Coherence } \\
\text { time }[\mathrm{ms}]\end{array}$ \\
\hline $2008-05-24$ & 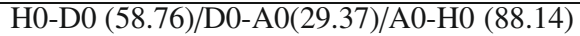 & $-99.8 /-99.8 /-99.8$ & $1.8731-2.4766$ & HD 169916 & 0.65 & 4.5 \\
\hline $2008-05-26$ & G1-D0 (71.55)/D0-H0(63.94)/H0-G1 (71.48) & $-46.0 / 70.5 / 7.0$ & $1.4492-2.3398$ & HD 166295 & 0.97 & 6.0 \\
\hline 2008-06-06 & H0-G0 (30.10)/G0-E0(15.06)/E0-H0 (45.17) & $-119.0 /-119.0 /-119.0$ & $1.4492-2.4458$ & $\begin{array}{l}\text { HD } 169916, \\
\text { HD } 146545\end{array}$ & 0.79 & 4.5 \\
\hline 2008-06-07 & H0-G0 (28.64)/G0-E0(14.3 & $-98.8 /-98.8 /-98.8$ & 2.5000 & HD 169916 & 1.16 & 2.0 \\
\hline 2008-06-08 & H0-G0 (31.98)/G0-E0(16.00)/E0-H0 (47.99) & $-108.0 /-108.0 /-108.0$ & $1.4454-2.3708$ & $\begin{array}{l}\text { HD } 169916, \\
\text { HD } 146545\end{array}$ & 0.60 & 2.5 \\
\hline 2008-07-03 & H0-G0 (29.63)/G0-E0(14.82)/E0-H0 (44.46) & $-100.0 /-100.0 /-100.0$ & $1.4578-2.2786$ & $\begin{array}{l}\text { HD } 169916, \\
\text { HD } 146545\end{array}$ & 1.29 & 2.0 \\
\hline 2008-07-04 & G1-DC & $-4.3 / 35.4$ & 1.445 & HD 169916 & 0.41 & 7.5 \\
\hline 2008-07-05 & $0-\mathrm{H} 0(63.92$ & $-46.1 / 63.9$ & -2.4832 & HD 169916 & 0.65 & 6.5 \\
\hline 2008-07-06 & G1-D0 (65.26)/D0-H0(55.29)/H0-G1 (71.48) & $-55.2 / 52.6 /-7.78$ & $1.4540-2.5211$ & HD 169916 & 0.60 & 3.5 \\
\hline
\end{tabular}
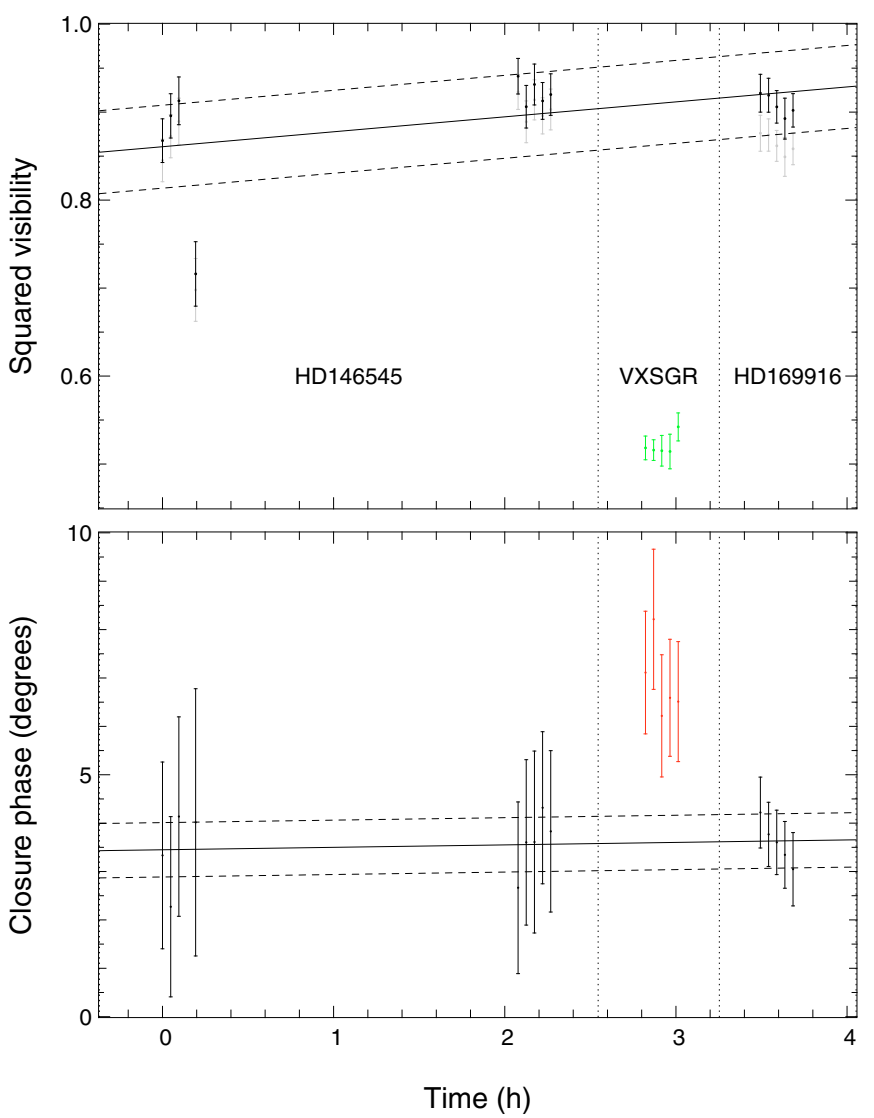

Fig. 1. Transfer function for the night 2008-07-03. Top panel: squared visibilities averaged over the region $2.1-2.2 \mu \mathrm{m}$ for both the calibration stars (gray with error bars) and the science star (green with error bars). The computed transfer function is approximated by a linear fit (solid black line), and dashed lines display the scatter. Bottom panel: closure phase averaged over the same spectral region for both the science target (red) and calibrators (black).

\section{Image reconstruction}

The first step in our analysis is a chromatic image reconstruction of our data to probe different layers in the photosphere and above. The image reconstruction process is similar to the one performed for T Lep in Le Bouquin et al. (2009). For the reconstruction, we used the MIRA software package (Thiébaut 2008; Cotton et al. 2008; Le Besnerais et al. 2008). The image was sought by minimizing a so-called cost function which is the sum

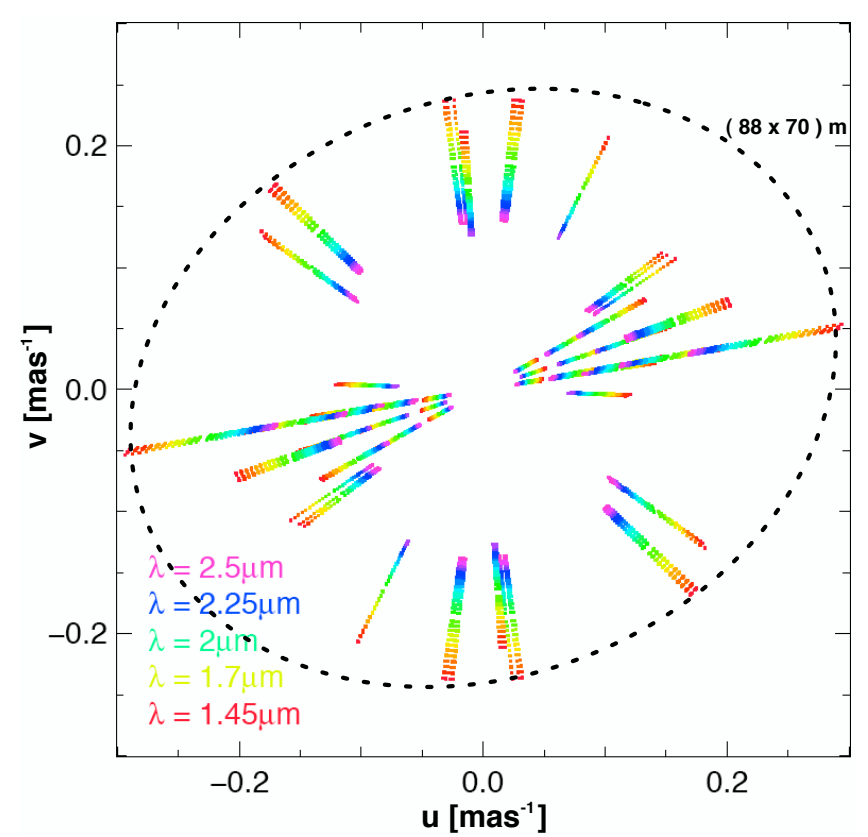

Fig. 2. UV-coverage for of the AMBER observations of VX Sgr. The radial extension of the uv tracks reflects the spectral coverage of our AMBER measurements, covering the $H$ and the $K$ bands.

of a regularization term plus data-related terms. The data terms enforce agreement of the model image with the measured data (visibilities). The regularization term is a $\chi^{2}$ minimization between the reconstructed image and an expected image. The expected image is issued from a preliminary image reconstruction, strongly constrained by the assumption of circularity. Each spectral bin has been processed independently (Fig. 3, left column). The reconstructed images clearly highlight different behaviors across the wavelength range: in the $\mathrm{H}$ band $(\approx 1.45-1.80 \mu \mathrm{m})$, the intensity distribution is inhomogeneous and a bifurcation of the image core into a few bright "spots" is visible. At $\approx 2.00 \mu \mathrm{m}$ and at the upper $K$ band edge $(\approx 2.35-2.50 \mu \mathrm{m})$ the radius appears extended and much larger than in the $H$ band. Artifacts may be introduced by the poor UV-plane coverage in one direction (north-west south-east, see Fig. 2). However, the detection of inhomogeneities is not in doubt because there are clear signatures in the closure phases (Fig. 1). 

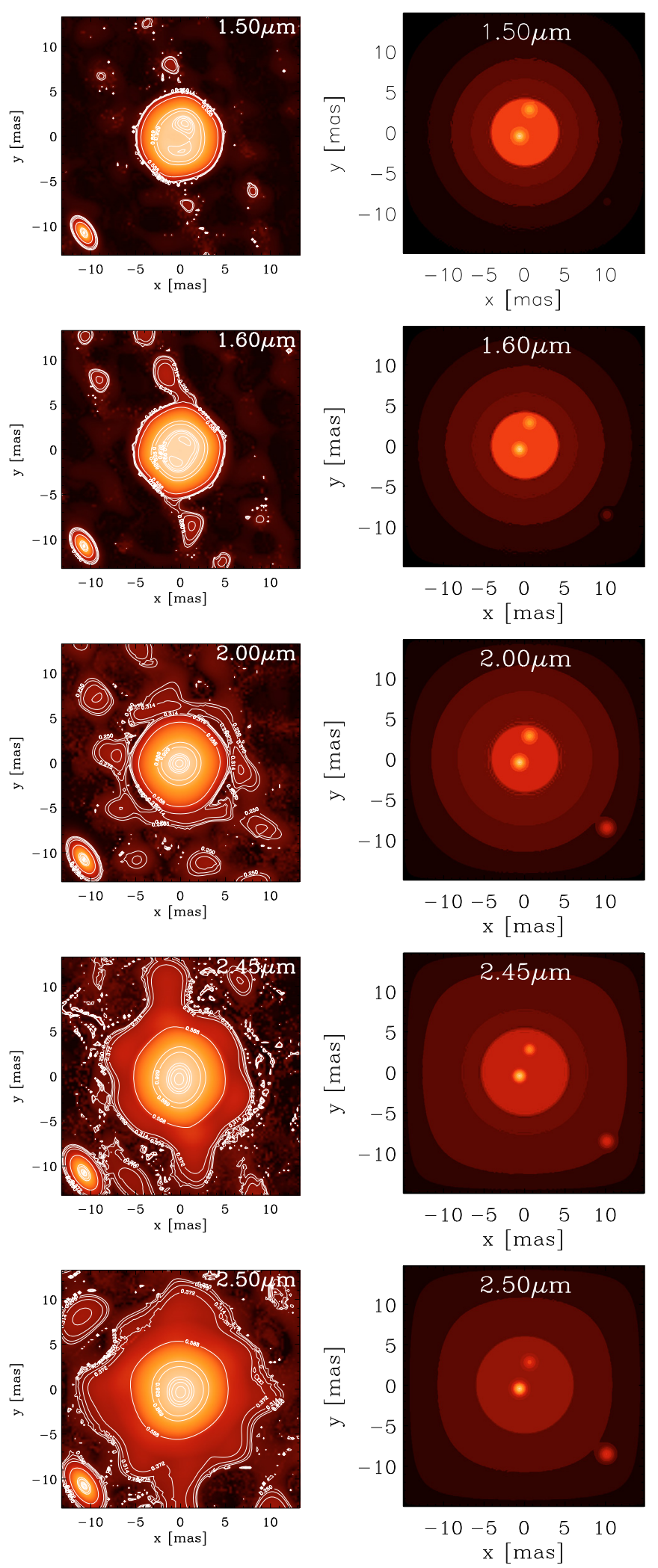

Fig. 3. Left column: reconstructed images of $\mathrm{VX}$ Sgr for several AMBER spectral bins across the $H$ and $K$ bands. The intensity, $I$, is normalized to the range $[0,1]$ and plotted as $I^{0.33}$. Some contour lines are indicated $(0.251,0.275,0.314,0.372,0.588,0.889,0.929,0.976,0.983$, $0.990,0.997)$. The resolution of the interferometer is illustrated in the bottom left part of each image by the PSF of an $88 \times 70 \mathrm{~m}$ telescope. Right column: images representing our best-fitting geometrical model for the same spectral channels. The intensity, $I$, is normalized to the range $[0,1]$ and plotted as $I^{0.33}$.

\section{Geometrical toy models}

The reconstructed images are not good enough to firmly establish inhomogeneities on VX Sgr's photosphere because the different sources of errors can smooth out the information. Thus, to confirm their presence and to constrain their flux relative to the total flux, we used a model-fitting approach. We approximated the star with a uniform disk and its extended shells by a Gaussian disk and added a series of point sources to model the spots. We performed a fit with all the observed wavelengths together, but used five regularly spaced reference wavelengths to effectively compute the model $(1.54,1.78,2.03,2.28$, and $2.52 \mu \mathrm{m})$. To match the data with the model, we interpolated the parameters for other wavelengths using cubic spline interpolation. The global optimum of the fit was found using a set of simulated annealing algorithms complemented by standard gradient descent algorithms (see Millour et al. 2009, for a first application of this approach). A model with three spots (i.e., performing a fit with a uniform disk, a Gaussian disk, and three spots) significantly enhance the fit $\left(\chi^{2} \approx 25\right)$, especially for the visibilities and closure phases up to spatial frequency of $\approx 240$ cycles/arcsec. Spatial frequencies above this value correspond to a single measurement at the largest baseline length. The details about the fits are reported in Table 2.

Our toy model probably does not perfectly describe the object at the highest angular resolution available, but it gives a reasonable fit to intermediate angular resolutions. We also note that two of the bright spots are located at the position of the stellar disk, and one has to be outside the stellar disk in order to fit the observed closure phases. We estimate that the upper value of the spots' flux is $\leq 10 \%$ of the total flux and that the flux of the spot located outside the photosphere very likely has a flux equal to zero in the $H$ band. Figure 3 (right column) displays the appearance of the toy model at some selected wavelengths. As a final remark, these images resemble the reconstructed images in the left column of the figure. We also note that the bright spot located outside the photosphere is particularly visible in the lower right part of each panel of the reconstructed images at lower wavelength and somehow less evident at longer wavelength even if it is expected by the geometrical model.

What we can conclude from this model-fitting approach is the following:

- a model with three spots can explain the visibilities and closure phases for spatial frequencies $\leq 240$ cycles/arcsec. Higher spatial frequencies are probed in our dataset by only one observation and indicate a more complicated object shape at smaller scales;

- the best-fitting model locates two of the spots inside the supposed photosphere of the star, but the third spot, mandatory for explaining the closure phase deviations, is located outside the photosphere and it is brighter at longer wavelengths;

- the spots have individual fluxes that, at all wavelengths, do not exceed $10 \%$ of the total flux of the star, for all wavelengths.

\section{Model fitting}

The visibility data of VX Sgr show significant wavelengthdependent features. We fitted the visibility curves and the closure phases using a Gaussian disk for each spectral channel, which gives more robust results than a uniform disk model. Figure 4 illustrates the change in apparent size with wavelength. This is consistent with what is visible in the reconstructed images 
Table 2. Parameters of the best geometrical toy model $\left(\chi^{2} \approx 25\right)$.

\begin{tabular}{|c|c|c|c|c|c|c|c|c|c|c|c|c|c|}
\hline \multirow{2}{*}{$\begin{array}{c}\lambda \\
{[\mu \mathrm{m}]}\end{array}$} & \multicolumn{2}{|c|}{ Gaussian disk } & \multicolumn{2}{|c|}{ Uniform disk } & \multicolumn{3}{|c|}{ Point source } & \multicolumn{3}{|c|}{ Point source } & \multicolumn{3}{|c|}{ Point source } \\
\hline & $\begin{array}{c}\text { size } \\
{[\mathrm{mas}]}\end{array}$ & $\begin{array}{l}\text { flux } \\
{[\%]}\end{array}$ & $\begin{array}{c}\text { size } \\
{[\mathrm{mas}]}\end{array}$ & $\begin{array}{l}\text { flux } \\
{[\%]}\end{array}$ & $\begin{array}{c}x \\
\text { [mas] }\end{array}$ & $\begin{array}{c}y \\
{[\mathrm{mas}]}\end{array}$ & $\begin{array}{l}\text { flux } \\
{[\%]}\end{array}$ & $\begin{array}{c}x \\
{[\mathrm{mas}]}\end{array}$ & $\begin{array}{c}y \\
{[\mathrm{mas}]}\end{array}$ & $\begin{array}{l}\text { flux } \\
{[\%]}\end{array}$ & $\begin{array}{c}x \\
{[\mathrm{mas}]}\end{array}$ & $\begin{array}{c}y \\
{[\mathrm{mas}]}\end{array}$ & $\begin{array}{l}\text { flux } \\
{[\%]}\end{array}$ \\
\hline 1.54 & $\leq 20$ & 26.7 & $8.3 \pm 2.5$ & 67.0 & $0.6 \pm 0.8$ & $2.8 \pm 0.5$ & $\leq 10$ & $-0.7 \pm 0.7$ & $-0.5 \pm 0.5$ & $\leq 10$ & $10 \pm 4$ & $-8.5 \pm 3.5$ & 0 \\
\hline 1.78 & $\leq 20$ & 32.1 & $8.1 \pm 1.5$ & 59.3 & - & - & $\leq 10$ & - & - & $\leq 10$ & - & - & 0 \\
\hline 2.03 & $\leq 20$ & 47.3 & $8.0 \pm 0.6$ & 43.8 & - & - & $\leq 10$ & - & - & $\leq 10$ & - & - & $\leq 5$ \\
\hline 2.28 & $\leq 30$ & 46.1 & $7.7 \pm 0.4$ & 47.5 & - & - & $\leq 10$ & - & - & $\leq 10$ & - & - & $\leq 10$ \\
\hline 2.52 & $28.7 \pm 26$ & 66.9 & $12.4 \pm 1.9$ & 22.9 & - & - & $\leq 10$ & - & - & $\leq 10$ & - & - & $\leq 10$ \\
\hline
\end{tabular}

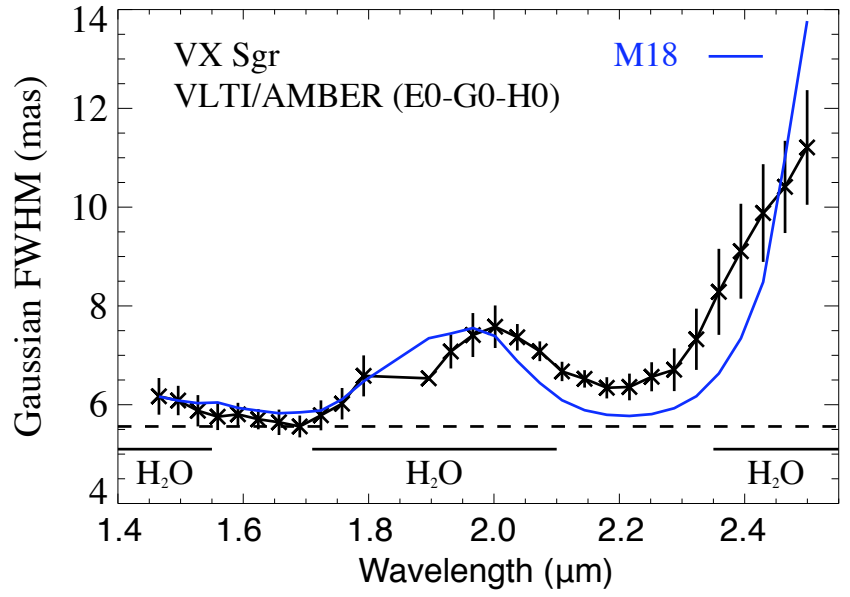

Fig. 4. VX Sgr Gaussian FWHM values as a function of the wavelength compared to the model M18 predictions. The dashed line indicates the smallest values. The position of the $\mathrm{H}_{2} \mathrm{O}$ bands are also indicated (after Lançon \& Wood 2000).

(Fig. 3): the radius gets larger between 1.8 and $2.1 \mu \mathrm{m}$, reaching local maximum at $2.0 \mu \mathrm{m}$, then gets smaller again to a minimum around 2.15 to $2.25 \mu \mathrm{m}$, and eventually becomes much larger toward $2.5 \mu \mathrm{m}$. However, Fig. 4 only gives a rough idea of the apparent diameter of VX Sgr because the intensity profile is not a Gaussian disk.

To reproduce the wavelength dependence, we used the onedimensional dynamic models of Ireland et al. (2004a,b) for oxygen-rich Mira stars that include the effect of molecular layers in the outer atmosphere. These are self-excited dynamic models whose gray atmospheric temperature stratification was recomputed on the basis of non-gray extinction coefficients that contain all relevant molecular absorbers (e.g., $\mathrm{H}_{2} \mathrm{O}, \mathrm{CO}$, TiO; solar abundances) but do not contain dust. The stellar parameters were assumed to be close to those of the M-type Mira prototypes o Cet and R Leo. This model series has been successfully used by Wittkowski et al. $(2007,2008)$ to explain AMBER observation of the Mira star S Ori. Because the VX Sgr stellar parameters are poorly understood, there is no fully consistent model of this star, and the models we used can only show typical characteristics of such a star. Figure 5 shows the comparison for short (left and central panels) and long (right panel) projected baselines. The wavelength dependence of the visibility is similar for all the nights.

For the fit, we followed the fitting procedure used by Wittkowski et al. (2008) and used only the short baseline data obtained with E0-G0-H0, because we do not expect to match details of the intensity profile probed at high spatial frequencies with the $\mathrm{M}$ series that has not been designed to match a
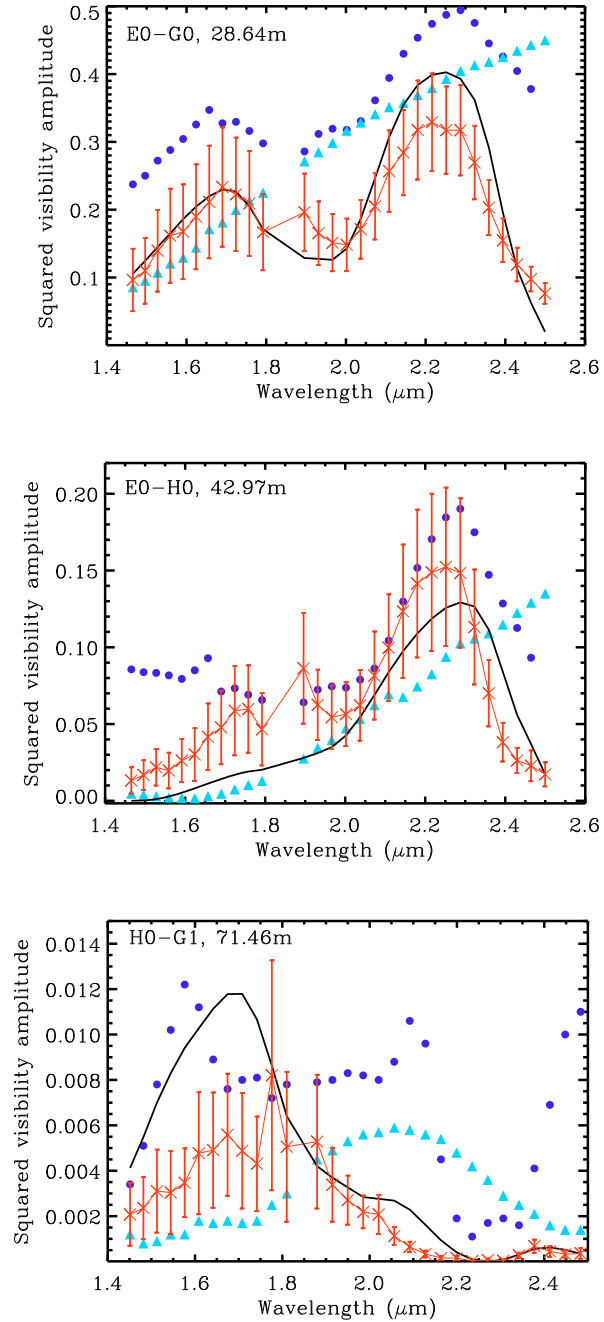

Fig. 5. Measured VX Sgr visibility data (red crosses with error bars) compared to the visibilities derived from (i) the 1D Mira atmosphere model M18 (model with a phase $\Phi=0.75$ ), black solid line; (ii) the best-matching synthetic image of the 3D simulation of an RSG star (top central and right panel in Fig. 6), light blue triangles; (iii) best-match synthetic images of the 3D simulation of an AGB star (bottom row in Fig. 6); blue dots. Left and central panels belong to the night 2008-0607 , and the right panel is for the night 2008-07-03.

star like VX Sgr. The dynamic models predict large changes in the monochromatic radius caused by the molecular layers above the continuum-forming region in the stellar photosphere. The bandpasses around 1.9 and $2.5 \mu \mathrm{m}$ are significantly affected by $\mathrm{H}_{2} \mathrm{O}$ molecules with some contribution of $\mathrm{CO}$ in the $H$ band and in the long-wavelength part $K$ band. The molecularband effects change significantly with phase as shown in 
Table 3. Parameters of the simulations, all with solar metallicity.

\begin{tabular}{ccccccc}
\hline \hline $\begin{array}{c}\text { Model } \\
\text { name }\end{array}$ & $\begin{array}{c}\text { Stellar } \\
\text { type }\end{array}$ & $\begin{array}{c}M \\
{\left[M_{\odot}\right]}\end{array}$ & $\begin{array}{c}L \\
{\left[L_{\odot}\right]}\end{array}$ & $\begin{array}{c}R \\
{\left[R_{\odot}\right]}\end{array}$ & $\begin{array}{c}T_{\text {eff }} \\
{[\mathrm{K}]}\end{array}$ & $\begin{array}{c}\log g \\
{[\mathrm{cgs}]}\end{array}$ \\
\hline M18 & Mira & 1.2 & 4840 & 210 & 3310 & - \\
st28gm06n06 $^{b}$ & AGB & 1 & 6935 & 429 & 2542 & -0.83 \\
st35gm03n07 $^{c}$ & RSG & 12 & 93000 & 832 & 3490 & -0.34 \\
\hline
\end{tabular}

Notes. ${ }^{(a)} 1 \mathrm{D}$ model; the radius is the Rosseland radius $R=0.81 R_{\mathrm{p}}$ at Mira phase $\approx 0.75$; (Ireland et al. 2004a); ${ }^{(b)} 3 \mathrm{D}$ model; Freytag \& Höfner (2008); ${ }^{(c)}$ 3D model; Chiavassa et al. (2009).

Wittkowski et al. (2008). The model M18 (see Table 3) provides the best fit to the VX Sgr data among the 20 available phase and cycle combinations of the $\mathrm{M}$ series.

The $\mathrm{M}$ model qualitatively explains the overall wavelength dependence of the apparent stellar radius (Fig. 5). However, while the fit is very good at short baselines, the comparison is less good at long baselines. Two reasons can explain this discrepancy: (i) the stellar parameters of the $\mathrm{M}$ models are not suited to VX Sgr; (ii) there are some surface inhomogeneities detected in the data, which are not in the $\mathrm{M}$ model. The angular photospheric diameter corresponding to the reference radius at $1.04 \mu \mathrm{m}$ is $\Theta_{\mathrm{M} 18}=8.82 \pm 0.43$ mas for short baselines where the fit is better. The error on the diameter includes systematic calibration and model uncertainties. This result agrees with what has been found by Monnier et al. (2004), $\Theta_{2.16 \mu \mathrm{m}}=8.7 \pm 0.4$ mas. However, our models do not include dust, while Monnier et al. find a dusty environment around the star with a flux contribution of about $20 \%$ in the $K$ band.

We also fitted Gaussian FWHM values to the synthetic visibility values based on the M18 model intensity profiles for each spectral channel, using the same procedure that was used to fit the measured data. The comparison between the fit to the M18 model intensity profiles and the fit to the measured data is shown in Fig. 4.

The available Mira models show good agreement with VX Sgr's data. However, this comparison can just give a basic qualitative picture of VX Sgr's surface, and more detailed interpretations of these data must be addressed to next generation models with stellar parameters appropriate for VX Sgr, which are currently not available.

\section{Complementary comparison with three-dimensional simulations}

In this section, we quantify whether the observed asymmetries are consistent with three-dimensional simulations of surface convection in RSG and AGB stars and whether their chaotic photospheric structure is adequate for explaining the different surface layers structures observed. The simulations are carried out with CO $^{5}$ BOLD (Freytag et al. 2002; Freytag \& Höfner 2008). Parameters of the models are given in Table 3. The pulsations are not artificially added to the models (e.g. by a piston) but are selfexcited. Excitation by unstationary sonic convective motions are responsible for the pulsations. Molecular opacities are taken into account, but radiation transport is treated in a gray approximation, ignoring radiation pressure and dust opacities. Dynamical pressure lets the density drop much more slowly than expected for a hydrostatic atmosphere. In our 3D simulations, the average density drops exponentially, and there is no sign of a wind or an extended shell with relatively high densities. Some more technical information can be found in Freytag \& Höfner (2008), the

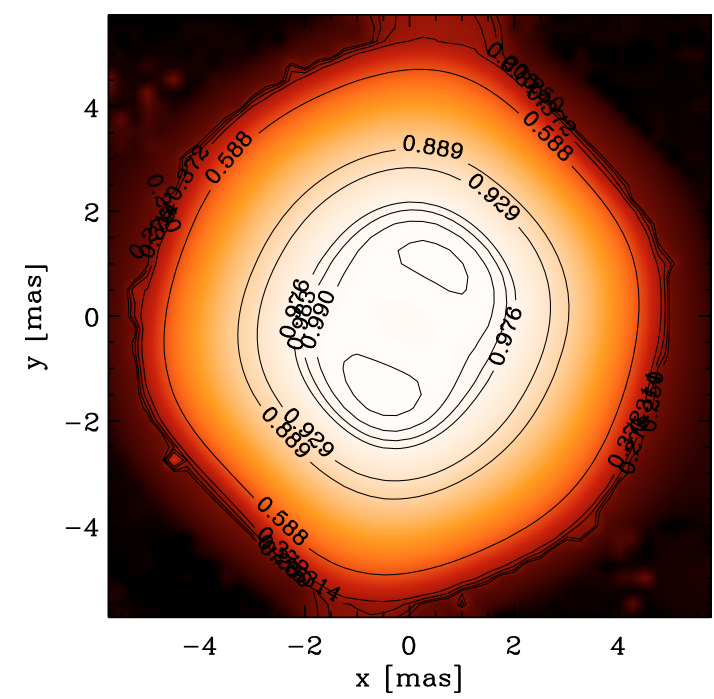

Fig. 6. Reconstructed image at $1.6 \mu \mathrm{m}$ with the intensity normalized to the range $[0,1]$ and plotted as $I^{0.33}$. The contour lines are the same as in Fig. 3.

$\mathrm{CO}^{5} \mathrm{BOLD}$ Online User $\mathrm{Manual}^{2}$, and in a forthcoming paper by Freytag (2010, in prep.).

We computed $\approx 3500$ synthetic images from these simulations at the same wavelengths as the observations using our 3D radiative transfer code OPTIM3D (Chiavassa et al. 2009). Then, we generated visibility curves using the method described by Chiavassa et al.. In the case of the RSG simulation, the synthetic images do not change strongly in diameter, shape, and asymmetries across the wavelength range of the observations, so that the observed wavelength dependence of the visibility is not explained by this approach (Fig. 5). The synthetic images of the AGB simulation show a noticeable wavelength dependence, and their agreement with the observed visibilities is slightly better than the RSG model, in particular at short baselines (left and central panel, blue dots of Fig. 5). Nevertheless, the two 3D simulations do not provide a better fit to our AMBER data than the Mira models described in Sect. 5.

At last, to explain the detected spots on the surface of the reconstructed images (Fig. 3), we compare the synthetic images corresponding to the best-fitting visibility curves of the 3D simulations (see Fig. 5) to the reconstructed image at $1.6 \mu \mathrm{m}$ (Fig. 6). This wavelength corresponds roughly to the $\mathrm{H}^{-1}$ continuous opacity minimum (i.e., the photosphere becomes more transparent). The reconstructed image shows two spots on the surface. Figure 7 displays the synthetic images of the RSG star, which show large convective cells $(\approx 4-5$ mas), and on top of that there are small-scale granules $(\approx 0.5-1$ mas). The observed visibility points do not show a good agreement especially at long baselines where the expected signal is much lower than the simulation's predictions.

The AGB star (Fig. 7) displays a pulsating stellar atmosphere with strong asymmetric structures caused by the shocks. The convective envelope is hardly visible because of the optical thickness of the atmosphere even without including the dust opacity tables (Freytag \& Höfner 2008). The AGB's visibilities and closure phases already show large departures from symmetry in the first lobe, but even in this case, the long baseline data points cannot be explained.

$\overline{2}$ www.astro.uu.se/ bf/co5bold_main.html 
A. Chiavassa et al.: VLTI/AMBER spectro-interferometric imaging of VX Sagittarii's inhomogenous outer atmosphere
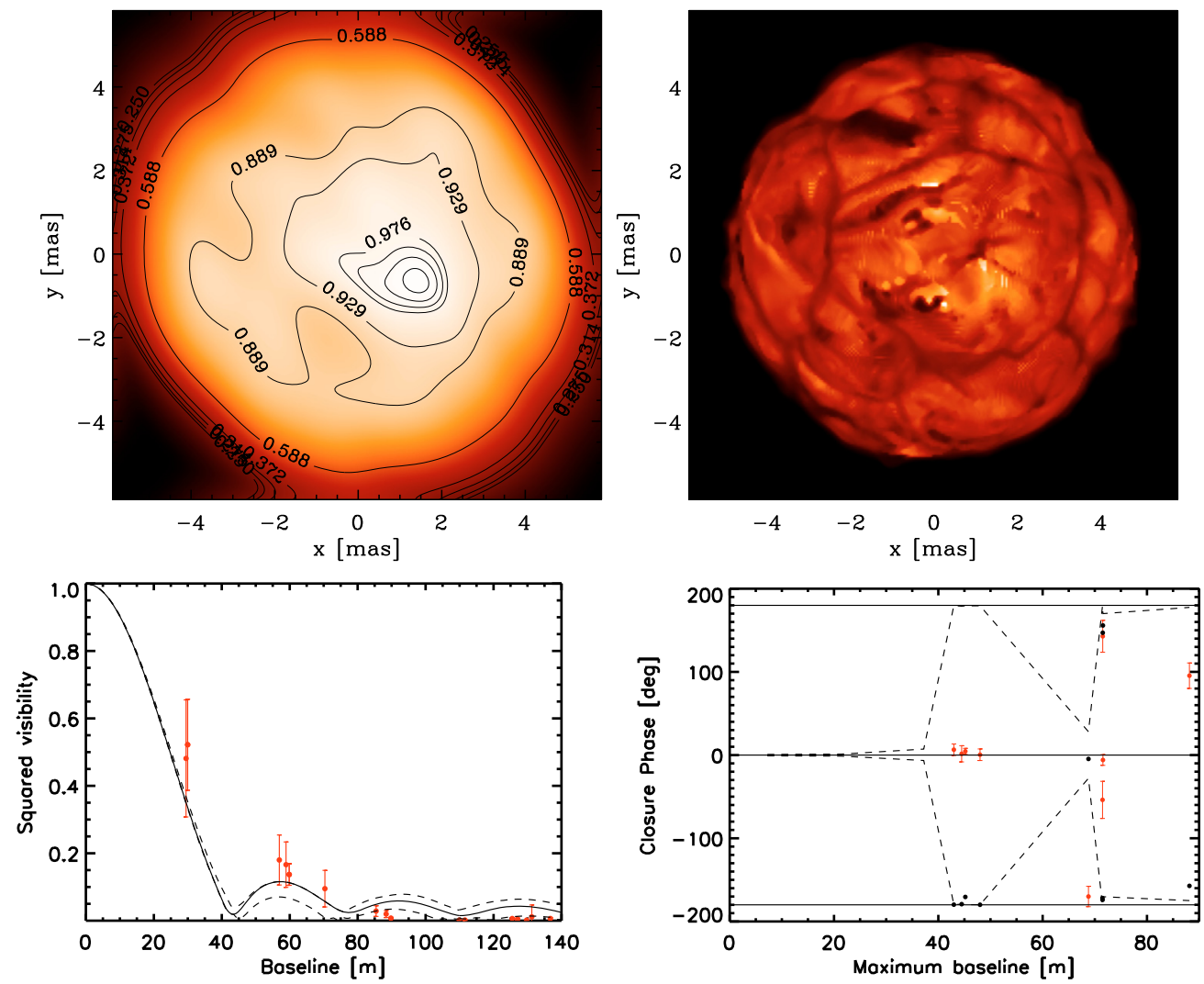

Fig. 7. Top left panel: synthetic image from the RSG simulation at $1.6 \mu \mathrm{m}$, convolved with a $5.9 \times 4.6$ mas PSF. The intensity is plotted as in Fig. 6 . The simulation has been scaled in size to approximately match the visibility data points. Top right panel: original RSG simulated image, the range is $[0 ; 300000] \mathrm{erg} \mathrm{cm}^{-2} \mathrm{~s}^{-1} \AA^{-1}$. Bottom panels: synthetic visibilities and closure phases derived from the above image (black solid line and dots) compared to the observations (red with error bars) at $1.6 \mu \mathrm{m}$. The dashed lines indicate the minimum and maximum amplitude of variations in the visibilities and closure phases issued from different rotations of the image. In bottom right panel, the axisymmetric case is represented by the solid lines.
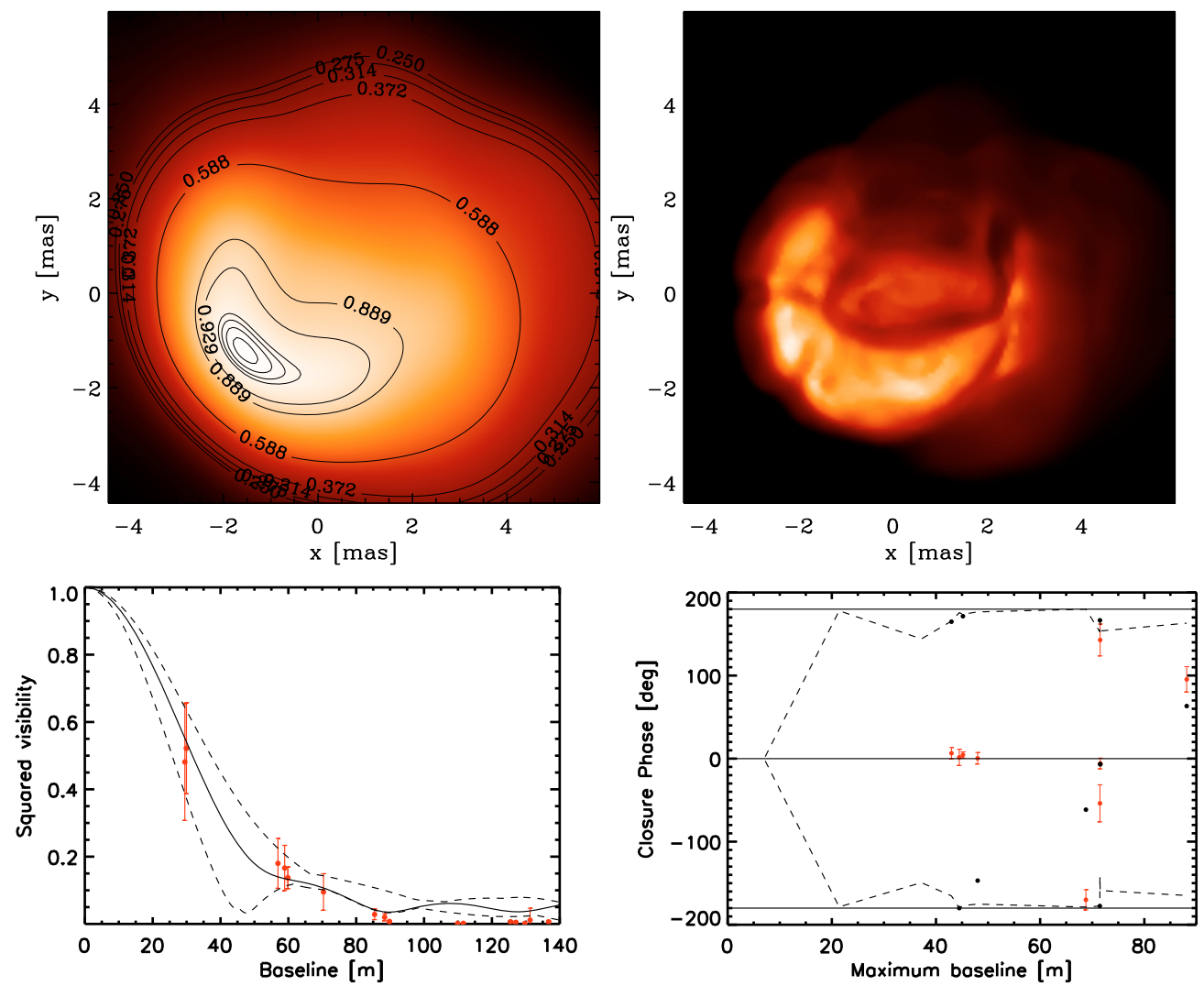

Fig. 8. Same as in Fig. 7 for the AGB simulation. Top right panel: original AGB image, the range is [0; 130000$] \mathrm{erg} \mathrm{cm}^{-2} \mathrm{~s}^{-1} \AA^{-1}$. 
That there are spots on the reconstructed image of VX Sgr can be qualitatively explained by the synthetic images, even if the distribution of the spots in our simulations has little chance of being exactly the one we observed with AMBER. Unfortunately, we cannot constrain the $3 \mathrm{D}$ simulations in terms of surface intensity contrast using these observations because we could not determine an accurate value for the spots' flux level (Table 2).

\section{Conclusions}

Our AMBER observations unveil, for the first time, the shape of VX Sgr's surface. The individual wavelength image reconstruction was carried out using the MIRA software. VX Sgr displays visibilities and images with strong wavelength dependence: (i) surface asymmetries in the $H$ band $(\approx 1.45-1.80 \mu \mathrm{m})$ and (ii) an extended radius at $\approx 2$ and $2.35-2.50 \mu \mathrm{m}$. We find that a geometrical toy model composed of a uniform disk plus a Gaussian disk and three spots gives a reasonable fit for the AMBER data. We claim that at least two spots are present on the photosphere of VX Sgr, and we show that this is qualitatively predicted by a 3D hydrodynamical simulations of stellar convection. In addition, the toy model shows that one spot resides outside the photosphere, and it is brighter at longer wavelengths. This spot's location outside the photosphere could be related to a complex and irregular structure in the surrounding of VX Sgr as already detected by Kervella et al. (2009) for the RSG alpha Ori. Also the two bright spots that appear at the position on the photosphere may both originate in the depths of the continuum photosphere or higher up in the molecular layers. In fact, these spots appear at all wavelengths (Fig. 3), also at the water bands, which hide a large part of the continuum photosphere. This might be a hint that they originate in the molecular layers far above the continuum photosphere.

We used 1D dynamical oxygen-rich Mira model predictions to explain the visibility data points, and we found that the fit is very good at short baselines. We conclude that $\mathrm{H}_{2} \mathrm{O}$ molecules strongly affect the visibility, making this molecule seem to be a dominant absorber in the molecular layers. The atmospheric structure of VX Sgr seems to qualitatively resemble Mira-star models, which show molecular layers above the continuum forming layer. In addition, its photosphere shows bright spots that can be related to giant-cell surface convection and possibly to the molecular layer. However, we must point out that the Mira model used here has stellar parameters that are not consistent with what we expect of VX Sgr, and there are only observations from a single epoch to do the interpretation. Because of various uncertainties, the classification of VX Sgr must be investigated further in the near future by first, combining multi-epoch photometric and spectroscopic observations to determine its stellar parameters better, and second, by observing with interferometers at higher spectral resolution to study the changes across the $H$ and $K$ bands in greater detail using new theoretical models with consistent stellar parameters.

Acknowledgements. We would like to thank the entire VLTI team and in particular J.-B. Le Bouquin. Moreover, we acknowledge John Monnier, Aldo Serenelli, Josef Hron, and Claudia Paladini for the enlightening discussions. A.C., B.P., and E.J. acknowledge financial support from ANR (ANR-06-BLAN-0105). We would like also to thank the JMMC team. The CNRS is acknowledged for supporting us with the Guaranteed Time Observations with AMBER. We thank the variable star observations from the AAVSO International Database contributed by observers worldwide and used in this research. We also thank CINES, France, and UPPMAX, Sweden, for providing the computational resources for the $3 \mathrm{D}$ simulations.

\section{References}

Bordé, P., Coudé du Foresto, V., Chagnon, G., \& Perrin, G. 2002, A\&A, 393, 183

Chapman, J. M., \& Cohen, R. J. 1986, MNRAS, 220, 513

Chen, X., Shen, Z.-Q., \& Xu, Y. 2007, Chin. J. Astron. Astrophys., 7, 531 Chiavassa, A., Plez, B., Josselin, E., \& Freytag, B. 2009, A\&A, 506, 1351

Cotton, W., Monnier, J., Baron, F., et al. 2008, Proc. SPIE, 7013, 48

Cutri, R. M., et al. 2003, The IRSA 2MASS All-Sky Point Source Catalog,

NASA/IPAC Infrared Science Archive, http://irsa.ipac.caltech. edu/applications/Gator/

Fawley, W. M. 1977, ApJ, 218, 181

Freytag, B., \& Höfner, S. 2008, A\&A, 483, 571

Freytag, B., Steffen, M., \& Dorch, B. 2002, Astron. Nachr., 323, 213

García-Hernández, D. A., García-Lario, P., Plez, B., et al. 2007, A\&A, 462, 711

García-Hernández, D. A., Manchado, A., Lambert, D. L., et al. 2009, ApJ, 705, L31

Haguenauer, P., Abuter, R., Alonso, J., et al. 2008, Proc. SPIE, 7013, 11

Heger, A., Jeannin, L., Langer, N., \& Baraffe, I. 1997, A\&A, 327, 224

Humphreys, R. M., Strecker, D. W., \& Ney, E. P. 1972, ApJ, 172, 75

Ireland, M. J., Scholz, M., Tuthill, P. G., \& Wood, P. R. 2004a, MNRAS, 355, 444

Ireland, M. J., Scholz, M., \& Wood, P. R. 2004b, MNRAS, 352, 318

Josselin, E., Loup, C., Omont, A., et al. 1998, A\&AS, 129, 45

Kamohara, R., Deguchi, S., Miyoshi, M., \& Shen, Z.-Q. 2005, PASJ, 57, 341

Kervella, P., Verhoelst, T., Ridgway, S. T., et al. 2009, A\&A, 504, 115

Kholopov, P. N., Samus', N. N., Kazarovets, E. V., \& Kireeva, N. N. 1987, Information Bulletin on Variable Stars, 3058, 1

Knapp, G. R., Sutin, B. M., Phillips, T. G., et al. 1989, ApJ, 336, 822

Lançon, A., \& Wood, P. R. 2000, A\&AS, 146, 217

Le Besnerais, G., Lacour, S., Mugnier, L. M., et al. 2008, IEEE Journal of Selected Topics in Signal Processing, 2, 767

Le Bouquin, J.-B., Abuter, R., Bauvir, B., et al. 2008, Proc. SPIE, 7013, 33

Le Bouquin, J.-B., Lacour, S., Renard, S., et al. 2009, A\&A, 496, L1

Levesque, E. M., Massey, P., Olsen, K. A. G., et al. 2005, ApJ, 628, 973

Levesque, E. M., Massey, P., Olsen, K. A. G., \& Plez, B. 2007, ApJ, 667, 202

Lockwood, G. W., \& Wing, R. F. 1982, MNRAS, 198, 385

Millour, F., Valat, B., Petrov, R. G., \& Vannier, M. 2008, Proc. SPIE, 7013, 132

Millour, F., Chesneau, O., Borges Fernandes, M., et al. 2009, A\&A, 507, 317

Monnier, J. D., Millan-Gabet, R., Tuthill, P. G., et al. 2004, ApJ, 605, 436

Murakawa, K., Yates, J. A., Richards, A. M. S., \& Cohen, R. J. 2003, MNRAS, 344, 1

Petrov, R. G., Malbet, F., Weigelt, G., et al. 2007, A\&A, 464, 1

Poelarends, A. J. T., Herwig, F., Langer, N., \& Heger, A. 2008, ApJ, 675, 614

Richichi, A., \& Percheron, I. 2002, A\&A, 386, 492

Richichi, A., Percheron, I., \& Khristoforova, M. 2005, A\&A, 431, 773

Schuster, M. T., Humphreys, R. M., \& Marengo, M. 2006, AJ, 131, 603

Sharpless, S. 1966, Spectral Classification and Multicolour Photometry, 24, 345

Siess, L. 2006, A\&A, 448, 717

Speck, A. K., Barlow, M. J., Sylvester, R. J., \& Hofmeister, A. M. 2000, A\&AS, 146,437

Tatulli, E., Millour, F., Chelli, A., et al. 2007, A\&A, 464, 29

Thiébaut, E. 2008, Proc. SPIE, 7013

van Leeuwen, F. 2007, A\&A, 474, 653

Vassiliadis, E., \& Wood, P. R. 1993, ApJ, 413, 641

Wallerstein, G. 1977, ApJ, 211, 170

Wittkowski, M., Boboltz, D. A., Ohnaka, K., Driebe, T., \& Scholz, M. 2007, A\&A, 470, 191

Wittkowski, M., Boboltz, D. A., Driebe, T., et al. 2008, A\&A, 479, L21 